may initially sound favourable for the procedure, the confidence interval for mortality is still wide enough to encompass no effect, while that of stroke is not.

With mortality now so low in STEMI trials, very large numbers of patients are required to reliably identify a clinically important improvement. The task requires massive multicentre trials or strategies that minimise per-patient costs by using established outcome-reporting infrastructure to focus on mortality, and perhaps introduce "retrospective consent".

\section{TRIPLE THERAPY ANTICOAGULATION FOLLOWING PERCUTANEOUS CORONARY INTERVENTION (PCI) WITH NOVEL ORAL ANTICOAGULANTS (NOAC) IS SAFE AND HAS NO ADVERSE EFFECTS ON BLEEDING POST PROCEDURE WHEN COMPARED TO TRIPLE THERAPY WITH VITAMIN K ANTAGONIST (VKA)}

${ }^{1}$ Reshma Amin* ${ }^{2}$ Nicholas McWilliams, ${ }^{2}$ Deacon Lee, ${ }^{2}$ Fatima Altaf, ${ }^{2}$ Farzana Virani, ${ }^{2}$ Katherine Dickinson, ${ }^{2}$ David Walker, ${ }^{3}$ Andrew Sharp, ${ }^{2}$ Robert Gerber. ${ }^{1}$ Kings NHS Trust; ${ }^{2}$ ESHT; ${ }^{3}$ Royal Devon and Exeter NHS Trust; *Presenting Author

\subsection{6/heartjnl-2016-309890.36}

Introduction Following PCI, dual antiplatelet therapy (DAPT) is indicated for prevention of stent thrombosis.

An increasing number of patients also require long term oral anticoagulation for stroke prevention in atrial fibrillation, for mechanical heart valves and venous thromboembolism.

Bleeding rates are historically higher in patients on triple therapy anticoagulation when compared to those on DAPT.

Since the introduction of NOAC, there are a variety of combinations of triple therapy anticoagulation that patients can be commenced on following PCI; however the bleeding risks between these groups are yet to be compared.

Methods We retrospectively studied 853 patients who underwent PCI in one centre from 2013-2014.

Of these, 103 patients required triple therapy, 49 with a Vitamin $\mathrm{K}$ antagonist (VKA) and 54 with NOAC.

The primary endpoint was 12 month bleeding complications as categorised by the Bleeding Academic Research Consortium (BARC). The secondary endpoint was major adverse cardiovascular and cardiac events (MACCE).

Results Of those on Triple Therapy Anticoagulation, 69\% of patients were male and $32 \%$ were aged 75 or above.

The indications for anticoagulation were $\mathrm{AF}$ (74\%), Venous Thromboembolism (12\%), left ventricular thrombus (13\%) and mechanical heart valve $(1 \%)$

Of those anticoagulated for AF, 95\% had a CHA2ds2-VASc score greater than 2 .

$29 \%$ of PCI was performed for NSTEACS and 17\% for ST segment elevation MI.

In the VKA category there was $12 \%$ minor bleeding compared to $22 \%$ in NOAC group (p0.0419).

There was no significant difference between TIMI Major and BARC bleeding between the two groups.

MACCE in the VKA group was $12 \%$ and in the NOAC group 5\% of which. $(\mathrm{p}=0.0412)$

Conclusion There is no significant difference in the bleeding risk between patients taking triple therapy anticoagulation following PCI with a NOAC or a VKA.

Patients requiring triple therapy anticoagulation following PCI have significantly less risk of MACCE at 12 month follow up with NOAC compared to VKA.

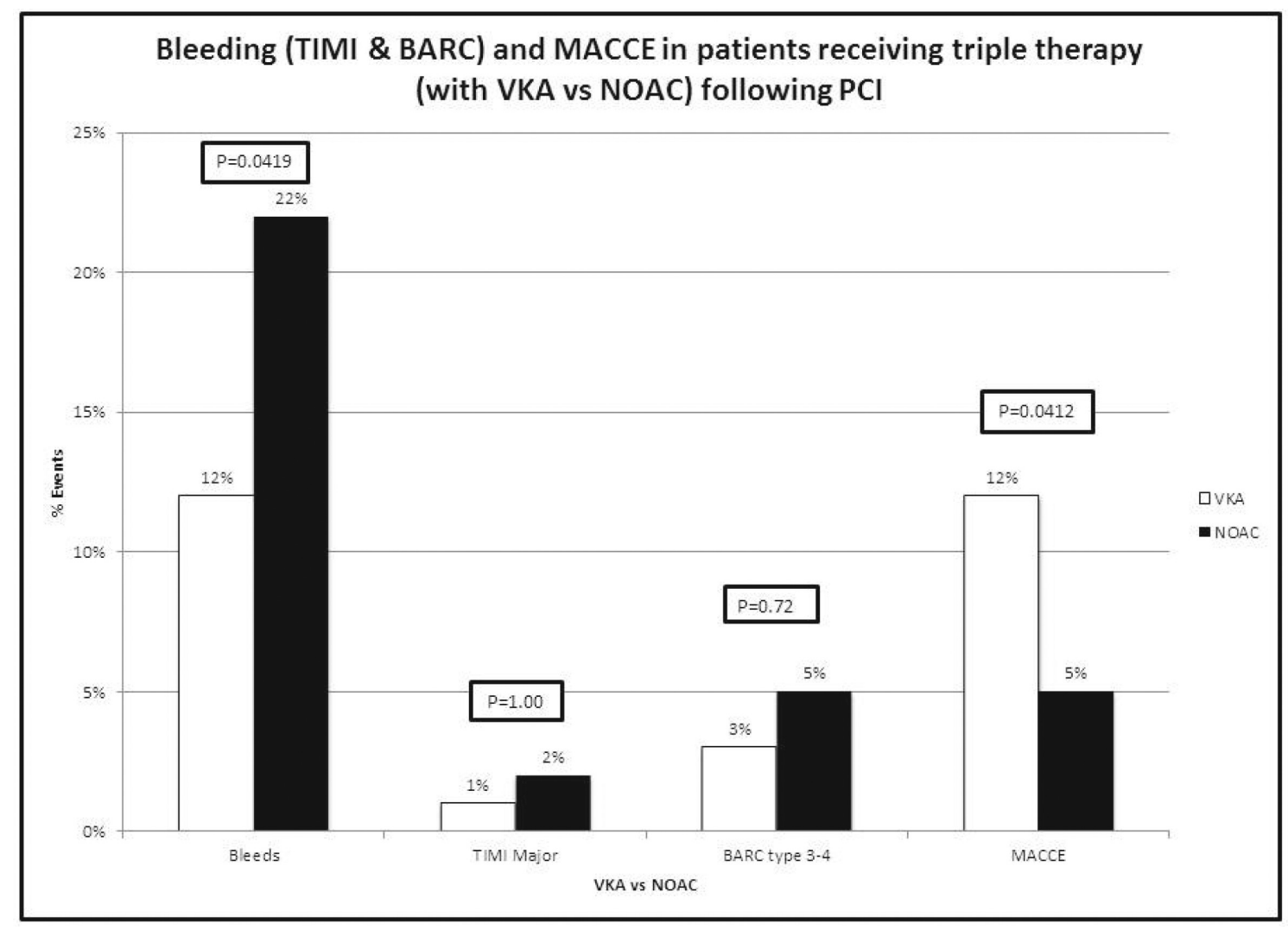

Abstract 36 Figure 1 Bleeding (TIMI \& BARC) and MACCE in patients receiving triple therapy (with VKA vs NOAC) following PCI 


\section{Imaging}

\section{TRANSOESOPHAGEAL ECHOCARDIOGRAPHY UNDERESTIMATES TRANSVALVULAR GRADIENTS FOLLOWING TRANS-CATHETER AORTIC VALVE IMPLANTATION (TAVI) - IMPLICATIONS FOR CLINICAL PRACTICE}

Andrea Solcanova*, Sarah Bates, Alison Calver, Nick Curzen, Dhrubo Rakhit, Benoy Shah. University Hospital Southampton; *Presenting Author

\subsection{6/heartjnl-2016-309890.37}

Background Trans-catheter aortic valve implantation (TAVI) is an effective treatment for high risk patients with severe aortic stenosis. As with all prosthetic valves, it is important to document accurate post-procedural gradients for future comparison. This study aimed to determine whether there was a difference between gradients measured immediately post-procedure by trans-oesophageal echocardiography (TOE) compared with the pre-discharge trans-thoracic echocardiogram (TTE). We also compared pre-TAVI gradients obtained by TTE vs. TOE.

Methods We used local and national databases to gather demographics on TAVI patients from our centre and to identify peak and mean aortic gradients measured by TTE and TOE prior to TAVI and also immediately following TAVI deployment (TOE) and prior to discharge (TTE). Data were compared using the paired t-test.

Results We identified 106 TAVI patients with complete echocardiographic data-sets. The mean age was $81+/-8$ yrs and 62 (54\%) were male. All patients received an Edwards Sapien valve. Pre-TAVI, there were no significant differences between TOE and TTE for both peak $(72.2+/-24.8 \mathrm{mmHg}$ vs $71.9+/-$ $24.0 \mathrm{mmHg}, \mathrm{p}=0.83)$ and mean $(41.4+/-15.0 \mathrm{mmHg}$ vs 42.4+/-14.9 mmHg,p $=0.22$ ) gradients. However, following TAVI, the peak trans-valvular gradients by TOE vs. TTE were $12+/-6 \mathrm{mmHg}$ vs $22+/-9 \mathrm{mmHg}(\mathrm{p}<0.001)$ and mean trans-valvular gradients were $6+/-3 \mathrm{mmHg}$ vs $11+/-5 \mathrm{mmHg}$ ( $\mathrm{p}<0.001)$. There were 36 patients with LV dysfunction: the results were unchanged after excluding these patients (peak gradient $12+/-6 \mathrm{mmHg}$ vs. $23+/-9 \mathrm{mmHg}, \mathrm{p}<0.001)$.

Conclusions Although TTE and TOE perform similarly prior to TAVI, the immediate post-procedural assessment of transaortic gradients by TOE leads to significant under-estimation compared to TTE. Intra-procedural TOE should not be used to define baseline peak and mean aortic gradients after TAVI.

\section{Valve Disease/Pericardial Disease/ Cardiomyopathy}

\section{MYOCARDIAL INFARCTION FOLLOWING SURGICAL AND TRANS-CATHETER AORTIC VALVE REPLACEMENT - IS PERI-PROCEDURAL REVASCULARISATION FOR TAVI NECESSARY?}

${ }^{1}$ Laura E Dobson", ${ }^{1}$ Tarique A Musa, ${ }^{1}$ Akhlaque Uddin, ${ }^{1}$ Timothy A Fairbairn, ${ }^{1}$ Peter P Swoboda, ${ }^{1}$ David P Ripley, ${ }^{1}$ Bara Erhayiem, ${ }^{1}$ Adam K McDiarmid, ${ }^{1}$ Pankaj Garg, ${ }^{2}$ Betsy Evans, ${ }^{2}$ Christopher J Malkin, ${ }^{2}$ Daniel J Blackman, ${ }^{1}$ Sven Plein, ${ }^{1} J o h n$ P Greenwood. ${ }^{1}$ University of Leeds; ${ }^{2}$ Leeds Teaching Hospitals NHS Trust; *Presenting Author

10.1136/heartjnl-2016-309890.38
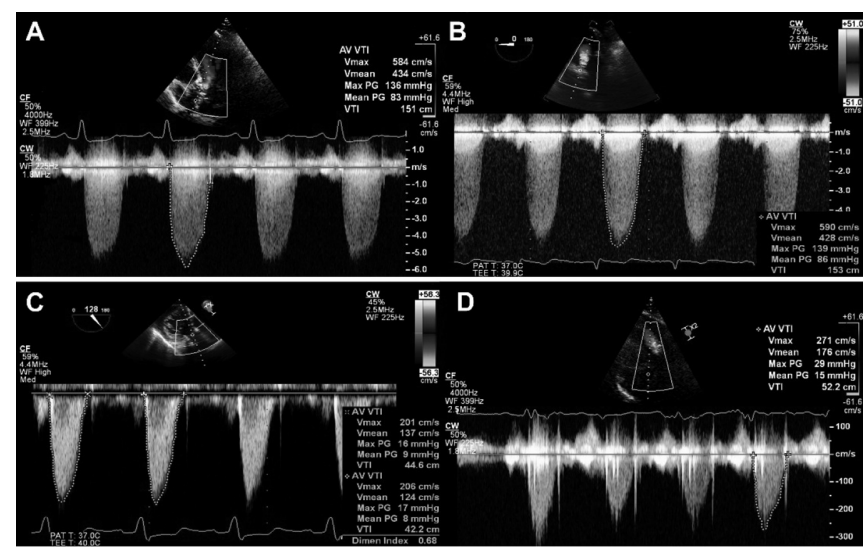

Abstract 37 Figure 1 Pre-TAVI TTE (A) vs TOE (B) and post-TAVI TOE (C) vs TTE (D)

Introduction Cardiac biomarker release is ubiquitous following surgical and trans-catheter aortic valve replacement (SAVR and TAVI), preventing accurate discrimination between release due to focal myocardial infarction (MI) and global myocardial injury (Figure 1). Furthermore, the need for peri-procedural revascularisation in TAVI is debated. Cardiovascular magnetic resonance (CMR) late gadolinium enhancement imaging (LGE) is the most sensitive imaging method to detect post-procedural new MI. Our study aimed to compare rates of new MI using CMR LGE before and $6 \mathrm{~m}$ after TAVI and SAVR.

Methods Ninety six patients with severe aortic stenosis undergoing TAVI $(\mathrm{n}=57)$ and SAVR $(\mathrm{n}=39)$ were prospectively recruited and identical scans performed prior to (median 1 day) and $6 \mathrm{~m}$ following aortic valve replacement. The presence of significant coronary artery disease (CAD) was determined by the occurrence of a $>50 \%$ stenosis in any major epicardial vessel. Areas of LGE were quantified with computer-assisted planimetry $\left(2 \mathrm{SD} ; \mathrm{cmr}^{42}\right.$, Circle CVI). Presence of new LGE was determined by direct comparison of pre and post-procedure scans.

Results The SAVR group was younger, less symptomatic, had less 3 vessel CAD and were at lower surgical risk than the TAVI group. Most (87\%) SAVR implants were bioprosthetic and most TAVI implants were Medtronic CoreValve (79\%) (86\% Transfemoral). Thirty-four (60\%) patients had non-revascularised CAD at the time of TAVI. MI pattern LGE was present at baseline in 24 TAVI (42\%) and 9 SAVR (24\%) patients. The rate of new MI was greater in the SAVR group than the TAVI group (SAVR, $\mathrm{n}=10(26 \%)$ vs. TAVI, $\mathrm{n}=3$ $(5 \%), p=0.004)$. Absolute mean mass of new MI was similar between groups (SAVR 1.1g $\pm 0.6 \mathrm{~g}$ vs. TAVI $2.0 \mathrm{~g} \pm 1.4 \mathrm{~g}$, $\mathrm{p}=0.395)$ as was infarct mass as a percentage of left ventricular mass (SAVR $1.0 \pm 0.4 \%$ vs. TAVI $2.2 \pm 1.3 \%, \mathrm{p}=$ 0.268 ) (Figure 2). None of the SAVR and only one of the TAVI infarcts were detected clinically (Figure 1 - blue dot). 34 patients $(60 \%)$ in the TAVI group had non-revascularised CAD at the time of TAVI, of whom only $3(9 \%)$ had new MI. In the SAVR group, 16 patients (41\%) underwent concurrent coronary artery bypass grafting (CABG). Patients undergoing $\mathrm{CABG}$ were less likely to have a new $\mathrm{MI}$ than those not requiring concurrent revascularisation (CABG 6.3\% vs. no CABG $39.1 \%, p=0.021)$. There was no difference in mean cardiopulmonary bypass time (New MI $88.5 \pm 31.1$ vs. No new MI $114.5 \pm 47.4 \mathrm{~min}, \mathrm{p}=0.112$ ) and aortic cross clamp 